\title{
Angekommen in der Kita? Zugang und Teilhabe schutzsuchender Kinder in Kindertagesbetreuung
}

\author{
Birgit Riedel/Christiane Meiner-Teubner
}

Die Bildung, Betreuung und Förderung in Einrichtungen der Kindertagesbetreuung wird als wichtiger Schlüssel für die Integration von Kindern mit Fluchterfahrung gesehen. In den Kitas begegnen sie gleichaltrigen einheimischen Kindern, schließen sie Freundschaften, werden sie mit den Gewohnheiten und kulturellen Werten des Landes vertraut und erlernen die deutsche Sprache. Gerade bei Kindern lässt sich beobachten, dass sie „erstaunlich schnell Deutsch lernen und sehr motiviert sind, sobald sie in eine Kita oder Schule gehen“. ${ }^{1}$ Zugleich kann die Kita Stabilität und Sicherheit vermitteln und den Kindern dabei helfen, belastende Erfahrungen von Ungewissheit, Angst, Fremdheit und Trauer zu bewältigen.

Trotz dieses großen Potenzials, das der Besuch einer Kindertageseinrichtung für die gesunde Entwicklung, Integration und Teilhabe von schutzsuchenden Kindern besitzt, spielte die Frage ihres Kita-Besuchs in der gesamten Integrationsdebatte und Flüchtlingsforschung eine untergeordnete Rolle. Fragen des Bildungszugangs und der Teilhabe wurden vor allem für ältere Kinder und Jugendliche mit dem Fokus auf Schule und Ausbildung thematisiert, selten jedoch für junge Kinder. Dies spiegelt sich auch in der begrenzten Datenlage wider, die bisher kaum quantifizierbare Aussagen zur frühen Bildung und Betreuung von schutzsuchenden Kindern zulässt. Zur relativen Unübersichtlichkeit der Situation trägt außerdem bei, dass erstens der Kita-Bereich anders als der Schulbereich dezentral, im Wesentlichen auf kommunaler Ebene, organisiert ist und sich durch eine Vielzahl von Trägern auszeichnet; ein Überblick über Strategien und Konzepte zum Umgang mit Kindern aus schutzsuchenden Familien ist - jenseits einzelner Landesprogramme - von daher kaum zu erhalten. Zweitens haben sich vor dem Hintergrund einer ohnehin knappen Platzsituation in Kindertageseinrichtungen Alternativkonzepte etabliert. So wurde und wird verschiedentlich versucht, Kinder aus schutzsuchenden Familien über niedrigschwellige Brücken- oder Einstiegsangebote zu erreichen, die jenseits des Regelsystems der Kindertagesbetreuung organisiert sind. Diese Angebote zeichnen sich ihrerseits durch eine große konzeptionelle Vielfalt aus, ein Überblick existiert auch hier nicht. Nicht zuletzt ist beim Blick auf die Datenlage zu berücksichtigen, dass anders als im Schulsystem der Kita-Besuch freiwillig ist, wodurch den Eltern als „Gatekeepern“ eine wichtige Rolle zukommt, die zugleich Kenntnisse und Wissen über Zugangs- und Finanzierungswege sowie Anmeldeprozeduren voraussetzt; aber auch die Präferenzen und Betreuungsvorstellungen der Eltern entscheiden letztlich über den Kita-Zugang der Kinder. Auch aus diesem Grund sind Betreuungsbedarfe und Zugangsmöglichkeiten von Kindern aus schutzsuchenden Familien nur schwer einzuschätzen.

Vor dem Hintergrund dieser komplexen Ausgangslage nähert sich der vorliegende Beitrag der im Titel gestellten Frage: Wie weit sind Kinder mit Fluchterfahrung in den Kitas angekom-

1 Lewek, M./Naber, A., Kindheit im Wartezustand. Studie zur Situation von Kindern und Jugendlichen in Flüchtlingsunterkünften in Deutschland. Deutsches Komitee für UNICEF, 2017, S. 37. 
men? Auf der Grundlage unterschiedlicher Datenquellen und Studien soll dargestellt werden, was wir über den Zugang und die Bildungsteilhabe dieser Kinder wissen: In welchem Umfang besuchen sie Angebote der Kindertagesbetreuung, welche Rahmenbedingungen finden geflüchtete Familien hinsichtlich des Zugangs vor, welche Hürden und Barrieren sind erkennbar und von welchen Erfahrungen berichten die Fachkräfte und Teams in den Einrichtungen, die schutzsuchende Kinder betreuen?

\section{Die Zahl der schutzsuchenden Kinder im Kita-Alter verzeichnet keinen Rück- gang}

Als die Zahl der Schutzsuchenden nach einem kontinuierlichen Anstieg in den Jahren 2015 und 2016 ihren Höhepunkt erreichte, befanden sich unter ihnen auch viele Familien mit kleinen Kindern, die ihre Heimat aufgrund von Krieg, Gewalt oder Verfolgung verlassen mussten. Etwa ein Drittel aller zu diesem Zeitpunkt nach Deutschland geflüchteten Menschen waren Kinder und Jugendliche, etwa die Hälfte unter ihnen jünger als sieben Jahre. Allein in diesen beiden Jahren wurden mehr als 180.000 Asylerstanträge für Kinder gestellt, die noch nicht das Schulalter erreicht hatten. Zwei Drittel dieser Kinder kamen aus Syrien, Afghanistan oder dem Irak. $^{2}$ Mittlerweile hat sich die Situation insofern geändert, als die Zahl der in Deutschland schutzsuchenden Menschen wieder deutlich zurückgegangen ist, allerdings immer noch über dem Niveau von 2014 liegt. Gleichzeitig steigt die Zahl der Kinder aus schutzsuchenden Familien, da in den - in den letzten Jahren hier angekommenen - Familien weitere Kinder geboren werden. Ablesen lässt sich dies unter anderem an der Zahl der unter 1-jährigen Kinder, für die Asylerstanträge gestellt werden. Sofern sich ihre Eltern noch im Asylverfahren befinden oder sich nur auf Grundlage einer ausländerrechtlichen Duldung hier aufhalten, müssen sie die Geburt eines Kindes melden und für das Neugeborene wird dann automatisch ein Asylerstantrag gestellt, der in die Statistik einfließt. Die Zahl dieser Anträge lag in den vergangenen Jahren bei $17.000 \mathrm{im}$ Jahr 2015, $34.000 \mathrm{im}$ Jahr 2016, 35.000 in 2017 und 16.000 im ersten Halbjahr 2018. Für 2017 und 2018 waren das fast 20\% aller gestellten Asylerstanträge - in den Vorjahren lag der entsprechende Anteil hingegen bei etwa 5\%.

Berechnet man auf der Grundlage der Asylantragsstatistik die Anzahl aller heute unter 7-jährigen Kinder, für die in den vergangen Jahren Asylerstanträge gestellt wurden, kommt man auf eine Gesamtzahl von mehr als 240.000 schutzsuchenden Kindern, die aktuell im Krippen- und Kindergartenalter sind. Damit ist allerdings nur eine Untergrenze markiert, da bei neugeborenen Kindern, deren Eltern bereits eine Aufenthalts- oder Niederlassungserlaubnis besitzen, die Entscheidung bei den Eltern liegt, ob ein Asylerstantrag für das Kind gestellt wird. Nicht zuletzt für die kürzlich geborenen Kinder wird es in den nächsten Jahren darum gehen müssen, ausreichend Plätze in der Kindertagesbetreuung zur Verfügung zu stellen.

Vor diesem Hintergrund erscheint zumindest erklärungsbedürftig, warum selbst im Feld der Kindertagesbetreuung die Debatte um die Integration von schutzsuchenden Kindern kaum noch geführt wird. Denn rein quantitativ bleibt die Frage des Kita-Zugangs von Kindern aus geflüchteten Familien in hohem Maß virulent, zumal derzeit viele Kinder in das Kindergartenalter „hineinwachsen“. Lässt sich darin ein Zeichen von Normalisierung erkennen und hat sich das Feld schlicht auf die neuen Gegebenheiten und diese neue Gruppe von Kindern eingestellt?

2 Bundesamt für Migration und Flüchtlinge (BAMF), Aktuelle Zahlen zu Asyl, Ausgabe Dezember 2017. 


\section{Anspruch auf einen Kita-Platz - rechtlich eine klare Sache?}

Geflüchtete Kinder haben wie alle anderen Kinder in Deutschland nach § 24 SGB VIII ab Vollendung des ersten Lebensjahres einen Rechtsanspruch auf einen Kita-Platz. Dafür müssen die Voraussetzungen des $\S 6$ Abs. 2 SGB VIII vorliegen, nämlich ein gewöhnlicher Aufenthalt nachgewiesen werden. Von Anfang an gab es jedoch unterschiedliche Auffassungen darüber, ab wann von einem gewöhnlichen Aufenthalt auszugehen ist. Anfang 2016 kam eine vom DJI in Auftrag gegebene Rechtsexpertise zu dem Ergebnis, dass ein gewöhnlicher Aufenthalt vorliegt, sobald die Kinder mit ihrer Familie nach Deutschland eingereist sind und nicht in ein anderes Land weiterziehen wollen. Demnach gilt der Rechtsanspruch auf einen Platz in der Kindertagesbetreuung für schutzsuchende Kinder unabhängig von ihrem Aufenthaltsstatus und ihrer Unterbringungsform ab dem Tag des Grenzübertritts. ${ }^{3}$ Die Bleibeperspektive der Kinder spielt juristisch keine Rolle. Davon abweichend wird in den meisten Bundesländern geflüchteten Kindern die Möglichkeit zum Besuch einer Kita oder Kindertagespflege jedoch erst eingeräumt, wenn die Familie die Erstaufnahmeeinrichtung verlassen hat und in der zugewiesenen Kommune in einer Anschlussunterkunft untergebracht ist. Dies sollte nach spätestens sechs Monaten der Fall sein - Erfahrungen zeigen, dass der Aufenthalt in einer Erstaufnahmeeinrichtung oder in vorübergehenden Notunterkünften für viele Kinder jedoch deutlich länger dauert. In der Praxis stellen sich noch weitere rechtliche und strukturelle Barrieren, die Aufmerksamkeit von Seiten der Forschung und Fachpolitik verdienen:

- So lässt sich erkennen, dass sich der Prozess des „Ankommens“ für geflüchtete Familien und ihre Kinder über einen langen Zeitraum erstrecken kann und von Vorläufigkeit, häufig erzwungenen Wohnortwechseln und Diskontinuität geprägt ist. Bis zur Etablierung eines dauerhaften Wohnsitzes können Jahre vergehen. Wie angesichts dieser Diskontinuitäten und Unplanbarkeit ein dauerhafter Kita-Besuch sichergestellt werden kann, ist fraglich. Es ist davon auszugehen, dass für viele schutzsuchende Kinder - sofern sie eine Kita besuchen Betreuungsabbrüche und Einrichtungswechsel häufig sind und sich die Frage des Zugangs zu einer Einrichtung öfter als einmal stellt.

- Besonders schwierig ist die Situation für Kinder mit „,geringer Bleibeperspektive“. In Aufnahme- und Ausweisungszentren („Ankerzentren“) werden Kinder sowohl rechtlich als auch räumlich von Teilhabemöglichkeiten, fairen Asylverfahren und essentiellen Kinderrechten wie dem Zugang zu Bildung ausgeschlossen. ${ }^{4}$ Trotz vielfacher Kritik wird in einigen Bundesländern an diesen Zentren festgehalten.

- Bestand 2015/16 Unsicherheit darüber, ab wann schutzsuchende Kinder einen Rechtsanspruch auf einen Betreuungsplatz haben, und führte dies häufig dazu, dass Kinder erst mit zeitlicher Verzögerung in Kitas aufgenommen wurden, so stellt sich heute demgegenüber die Frage, inwieweit ein negativer Asylbescheid und die Verhängung einer Ausreisepflicht möglicherweise einen Hinderungsgrund darstellen, dass Kinder aus geflüchteten Familien in einer Kita angemeldet oder aufgenommen werden.

3 Meysen, T./Beckmann, J./González Méndez de Vigo, N., Flüchtlingskinder und ihre Förderung in Tageseinrichtungen und Kindertagespflege. Rechtsexpertise im Auftrag des Deutschen Jugendinstituts, 2016.

4 Lewek/Naber (Anm. 1), S. 37. 
- Nicht zuletzt haben bisherige Studien zu dem Thema auf das Problem des Platzmangels hingewiesen, das sich in vielen Großstädten - und hier nicht nur mit Blick auf unter 3-jährige Kinder - zeigt. Wo jedoch Plätze knapp sind, ist die Wahrscheinlichkeit für Kinder aus schutzsuchenden Familien besonders hoch, dass sie sich in der „Warteschleife auf einen Kita-Platz" wiederfinden. ${ }^{5}$

\section{Vorsichtig positive Bilanz: Kinder kommen in der Fläche an}

Bis heute liegen keine verlässlichen bundesweiten Zahlen dazu vor, wie weit der Rechtsanspruch eingelöst wird und wie viele Kinder aus schutzsuchenden Familien in den vergangenen Jahren in Kindertageseinrichtungen aufgenommen wurden bzw. alternative Angebote besuchen. Allerdings wurde verschiedentlich versucht, die Größenordnung näherungsweise zu bestimmen.

Der Bildungsbericht versucht eine Annäherung über den jüngsten Zuwachs an Kindern mit einer anderen Familiensprache als Deutsch, die in Kindertageseinrichtungen betreut werden. ${ }^{6}$ Er kann sich dabei auf die Kinder- und Jugendhilfestatistik und damit eine bundesweite Vollerhebung in Kindertagesbetreuungsangeboten stützen. Die Kinder- und Jugendhilfestatistik weist generell Kinder mit Migrationshintergrund und mit nicht-deutscher Familiensprache aus, das Merkmal Flucht wird jedoch nicht spezifisch erhoben. Somit ist zu berücksichtigen, dass beim Zuwachs der letzten Jahre bei den Kindern mit nicht-deutscher Familiensprache auch die innereuropäische Migration ins Gewicht fallen dürfte. Der Anstieg ist immerhin bemerkenswert: Waren es 2007 noch rund 366.000 Kinder mit Migrationshintergrund in Kindertagesbetreuung, die zuhause vorrangig eine andere Sprache als Deutsch sprachen, so sind es 2017 fast 563.000, also 54\% mehr. Allein zwischen 2015 und 2017 - also während und kurz nach der starken Neuzuwanderung - ist die Anzahl dieser Kinder um mehr als 75.000 angestiegen. 2015 und 2016 hat zugleich eine hohe EU-Binnenwanderung stattgefunden. Unter der Annahme, dass sich beide Gruppen mit Blick auf die Kita-Nutzung ähnlich verhalten, errechnet der Bildungsbericht für 2017, dass der Anteil der Kinder aus schutzsuchenden Familien, die Kindertagesbetreuungsangebote nutzen, an allen Kindern mit Fluchthintergrund bei den unter 3-Jährigen bei $17 \%$ und bei den 3- bis 5-Jährigen bei 42\% liegen könnte. Er weist dies allerdings als „Maximalvariante“ aus. ${ }^{7}$ Erste Analysen der Kinder- und Jugendhilfestatistik für das Jahr 2018 zeigen, dass die Anzahl der Kinder mit Migrationshintergrund und nicht-deutscher Familiensprache erneut um rund 30.000 Kinder gestiegen ist, was darauf hindeutet, dass weitere Kinder aus schutzsuchenden Familien in Kindertagesbetreuungsangebote aufgenommen wurden und sich damit auch der Anteil der Kinder, die diese Angebote besuchen - zumindest leicht - erhöht haben dürfte.

$\mathrm{Zu}$ einer höheren Beteiligungsquote kommt eine Erhebung von IAB, BAMF und SOEP unter Geflüchteten, die zwischen dem 1.1.2013 und dem 31.1.2016 nach Deutschland gekommen sind. Während die Studie für unter 3-jährige Kinder eine ähnliche Quote (15\%) ausweist wie

5 Meiner-Teubner, C., Flüchtlingskinder in der Warteschleife, DJI-Impulse 114 (3), 2016, S. 19.

6 Autorengruppe Bildungsberichterstattung, Bildung in Deutschland. Ein indikatorengestützter Bericht mit einer Analyse zur Wirkungen und Erträgen von Bildung, 2018.

7 Ebd., S. 75. 
der Bildungsbericht, liegt ihr zufolge der Kita-Besuch bei den 3- bis unter 6-jährigen Kindern deutlich höher, nämlich bei $80 \% .{ }^{8}$ Hier ist allerdings einschränkend anzumerken, dass die Auswahl der Befragten hoch selektiv ist - es handelt sich um schutzsuchende Familien mit hoher „Bleibeperspektive“, die sich zum Teil schon länger in Deutschland aufhalten und in der Mehrzahl bereits über einen Aufenthaltstitel verfügen. Insofern ist diese Gruppe nicht repräsentativ für die Mehrheit der in 2015/16 in großer Zahl hinzugekommenen Schutzsuchenden. Darüber hinaus wurde danach gefragt, ob die Kinder dieser Familien (nicht näher spezifizierte) Kindertagesbetreuungsangebote nutzen. Es lässt sich vermuten, dass dieser Begriff von den Befragten unterschiedlich aufgefasst wurde und sich nicht allein auf Kindertageseinrichtungen beschränkt, sondern tendenziell jede Form der Betreuung wie Spielgruppen, stundenweise Angebote durch Ehrenamtliche usw. mit einschließt. Mit Sicherheit stellt die sehr hohe Beteiligungsquote speziell der 3- bis unter 6-Jährigen daher eine Überschätzung des Kita-Besuchs dar. Als positive Botschaft kann jedoch aus dem Ergebnis herausgelesen werden, dass mit einer längeren Aufenthaltsdauer der Zugang zu Einrichtungen der Kindertagesbetreuung oder gegebenenfalls anderen außerfamiliären Betreuungsangeboten in höherem Maße gelingt. Dies wird auch durch Regressionsanalysen bestätigt, die spezifisch für 3- bis 6-jährige Kinder zeigen, „dass neben dem Alter und der Region, in der die Kinder leben, auch die Dauer des Aufenthalts in Deutschland sowie die Art der Unterkunft weitere Faktoren sind, die statistisch signifikant positiv mit der KitaNutzung zusammenhängen. Insbesondere geflüchtete Kinder dieser Altersgruppe, die in privaten Wohnungen statt in Gemeinschaftsunterkünften leben, die bereits länger in Deutschland sind und die in Westdeutschland leben, haben eine höhere Wahrscheinlichkeit, eine Kita zu nutzen“.9 Bei unter 3-jährigen Kindern haben hingegen nur das Alter sowie der Aufenthalt in Ostdeutschland einen signifikant positiven Einfluss auf einen Kita-Besuch.

Einen anderen Versuch der Annäherung stellen Studien dar, die sich an Kindertageseinrichtungen richteten und diese danach befragten, ob und in welchem Umfang sie schutzsuchende Kinder betreuten. Hier konnte eine bundesweite DJI-Kitabefragung zu Beginn des Jahres 2016 zeigen, dass von den knapp 1.800 Kindertageseinrichtungen, die sich an der Befragung beteiligten, gut jede dritte (36\%) zu diesem Zeitpunkt bereits Kinder aus schutzsuchenden Familien aufgenommen hatte. ${ }^{10}$ Überwiegend wurden dabei 1 bis 2 schutzsuchende Kinder im Kindergartenalter in die bestehenden Gruppen integriert. Bemerkenswert daran war, dass sich infolge der landesinternen Verteilungsmechanismen die Aufnahme, Integration und Betreuung geflüchteter Kinder nicht nur auf „migrationserprobte“ Kitas in den Großstädten konzentrierte, sondern überproportional häufig von Kindertageseinrichtungen in den Klein- und mittelgroßen Städten sowie den Landgemeinden geleistet wurde, für die diese Erfahrung (Familien mit anderem kulturellem Hintergrund, Kinder, die zunächst kein Deutsch sprechen) in vielen Fällen ganz neu war und auch mit einiger Verunsicherung einherging. Die Studie zog aus dem Ergebnis zunächst die vorsichtig positive Bilanz, dass schutzsuchende Kinder offenbar in der Fläche

8 Gambaro, L./Liebau, E./Peter, F./Weinhardt, F., Viele Kinder von Geflüchteten besuchen eine Kita oder Grundschule - Nachholbedarf bei den unter Dreijährigen und der Sprachförderung von Schulkindern. DIW Wochenbericht 19, 2017, S. 379.

9 Ebd., S. 385.

10 Baisch, B., et al.., Flüchtlingskinder in Kindertagesbetreuung: Ergebnisse der DJI-Kita-Befragung „Flüchtlingskinder“ zu Rahmenbedingungen und Praxis im Frühjahr 2016, 2016. Zu einem ganz ähnlichen Ergebnis kam eine im Frühsommer desselben Jahres durchgeführte bayernweite Kita-Befragung von Lorenz, S./Wertfein, M., Flüchtlingskinder in Kindertageseinrichtungen. 11 zentrale Befunde, IFPStudie zur Aufnahme von Kindern mit Fluchterfahrung in bayerischen Kitas, 2017. 
in den Kitas angekommen sind - und dies obwohl politische oder verbandliche Strategien und Vorgaben meist fehlten und dieses Ergebnis im Wesentlichen auf das große Engagement von Ehrenamtlichen sowie Fachkräften im Feld der Kindertagesbetreuung zurückgeführt wurde. ${ }^{11}$

Gleichzeitig machte die Studie auf bestehende Zugangshürden aufmerksam. Nach Aussage der Kindertageseinrichtungen verhinderte vielerorts der Platzmangel die Aufnahme von geflüchteten Kindern. Jede zweite Kita gab an, dass sie keine freien Plätze hat, wobei dieser Grund besonders in den Großstädten zu Buche schlug - und zwar nicht nur im u3-Bereich, sondern auch bei Kindern ab drei Jahren. Das führt vielfach auch dazu, dass die Kinder nicht zu dem Zeitpunkt in die Kitas aufgenommen werden können, ab dem ein Bedarf angemeldet wird, sondern erst zu Kita-Jahresbeginn, wenn Plätze frei werden, weil ältere Kinder in die Schule wechseln. ${ }^{12}$ Angesichts eines weiterhin stark steigenden Platzbedarfs in den nächsten Jahren ist nicht so bald mit einer Entspannung dieser Situation zu rechnen. ${ }^{13}$

Auch konnte mit der Studie gezeigt werden, dass es in der Regel intervenierende Personen braucht, um den Weg in die Kita zu ebnen, da den Eltern meist die Rechts- und Institutionenkenntnis fehlt, um allein einen Kita-Platz zu finden, die Anmeldeprozeduren zu meistern und die Finanzierung $\mathrm{zu}$ regeln. Insbesondere ehrenamtlichen HelferInnen kommt dabei eine Schlüsselrolle zu. Vor allem in ländlichen Gebieten, Klein- und Mittelstädten sind sie die wesentlichen Wegbereiter, die geflüchtete Familien über das Angebot der Kindertagesbetreuung aufklären und sie bei der Anmeldung begleiten bzw. diese initiieren ${ }^{14}$ - oder wie eine Studie des IFP plastisch illustriert: ${ }^{15}$ „Den Besuch der Kita mit den Familien genau vorstrukturieren und wiederholt motivieren, an vereinbarte Besichtigungs-/Besuchstermine immer wieder erinnern, mit ihnen zusammen den Fußweg zu den Kitas gehen, Unterlagen ausfüllen bzw. zusammenstellen - dieses und manches mehr hilft den Familien, auch tatsächlich das Betreuungsangebot der Kitas wahrnehmen zu können. Grundvoraussetzung ist allerdings, dass Unterkünfte und Kitas in erreichbarer Distanz liegen."

Jenseits von notwendigen Zugangserleichterungen (muttersprachliche Informationen und Formulare, flexible Anmeldefristen, Hilfe bei der Beantragung von Kostenübernahmen bspw. für die Elternbeiträge oder die Mittagsverpflegung) verdienen zwei weitere Aspekte Beachtung:

- So wurde insbesondere zum Höhepunkt des Zuzugs von Schutzsuchenden aus dem Praxisfeld berichtet, dass manche der geflüchteten Kinder nur sehr kurz in einer Einrichtung verweilten. Die bayernweite Studie des IFP konnte zeigen, dass von den schutzsuchenden Kindern, die die Kita zum Zeitpunkt der Befragung bereits wieder verlassen hatten, etwas mehr als die Hälfte $(54,3 \%)$ die Einrichtung länger als 6 Monate, ein gutes Viertel sogar länger als ein Jahr besuchte; 45,7\% der aufgenommenen Kinder blieben allerdings maximal ein halbes Jahr in der Kita. ${ }^{16}$ Als Abmeldegrund wurde von den Kitas mit 40\% die Zuweisung der Familien zu einer anderen Unterkunft am häufigsten genannt, gefolgt von Abschiebungen

11 Riedel, B./Lüders, K., Flüchtlingskinder in der Kita - Zugänge gestalten, Kooperationen stützen! Kinderund Jugendschutz in Wissenschaft und Praxis, 61 (4), S. 138.

12 Kopp, K./Meiner-Teubner, Ch./Pothmann, J., Schutz- und asylsuchende junge Menschen, in: Autorengruppe Arbeitsstelle Kinder- und Jugendhilfestatistik, Kinder- und Jugendhilfereport 2018.

13 Autorengruppe Bildungsberichterstattung (Anm. 6), S. 68.

14 Baisch et al. (Anm. 10).

15 Lorenz, S./Wertfein, M. (Anm. 10), S. 68.

16 Ebd., S. 25. 
(28\%). Inwiefern sich darin die spezifische Situation 2015/16 widerspiegelt, lässt sich hier nicht abschließend klären.

- Ebenfalls kaum etwas ist bisher darüber bekannt, inwiefern Vorbehalte auf Seiten der geflüchteten Familien einen Kita-Besuch verhindern bzw. zu Abmeldungen führen. Vielfach wird in diesem Zusammenhang auf andere Betreuungstraditionen in den Heimatländern der Geflüchteten verwiesen und es wird vermutet, dass es den Eltern möglicherweise schwerfällt, nach dem Erlebnis der Flucht sich von ihrem Kind zu trennen und dieses einer fremden Institution anzuvertrauen. In diesem Zusammenhang wird häufig argumentiert, dass schutzsuchende Familien eher andere Formen der Betreuung benötigen, z.B. Eltern-Kind-Angebote, die die Eltern einbeziehen, oder dass sie über niedrigschwellige Angebote erst an die Kita „herangeführt“" werden müssen. An dieses Argument knüpfen unter anderem das Bundesprogramm „Kita-Einstieg“, das NRW-Landesprogramm zu sogenannten „Brückenangeboten“, aber auch zahlreiche Projekte auf Kommunalebene an. Im Rahmen des BMFSFJ-Programms „Kita-Einstieg: Brücken bauen in frühe Bildung“ etwa werden bundesweit an rund 150 Standorten niedrigschwellige Angebote gefördert, die über die Arbeit in Kitas informieren und den Zugang vorbereiten und unterstützend begleiten. Das NRW-Programm „Brückenangebote“ unterstützt ebenfalls niedrigschwellige, pädagogische Angebote, die Kinder mit Fluchthintergrund und ihre Eltern adressieren wie Eltern-Kind-Angebote, Spielgruppen oder mobile Angebote. Allein zwischen 2015 und 2016 sind rund 1.100 Maßnahmen, in denen knapp 10.000 Kinder betreut werden konnten, bewilligt worden. ${ }^{17}$

Trotz der wichtigen Rolle dieser Angebote sollte allerdings das Ziel, schutzsuchende Kinder in Regelkitas zu integrieren und dort gemeinsam mit einheimischen Kindern zu fördern und $\mathrm{zu}$ betreuen, nicht aus dem Blick geraten. Auch ist bei pauschalierenden Annahmen über Vorbehalte der Eltern gegenüber Kitas Vorsicht geboten. Aus eigener Erfahrung zeigen geflüchtete Familien oft ein großes Interesse daran, dass ihre Kinder frühzeitig Deutsch lernen, und ist ihnen die Bedeutung eines regelmäßigen Kita-Besuchs hierfür bewusst.

\section{Erfahrungen bei der Betreuung von Kindern aus schutzsuchenden Familien}

Im Feld der Kindertagesbetreuung hat der Zuzug von schutzsuchenden Familien mit jungen Kindern eine Welle der Solidarität hervorgerufen. Generell war zu beobachten, dass in den Jahren 2015/16 auf der einen Seite bei den Kita-Fachkräften und Trägern eine erhebliche Verunsicherung darüber bestand, welche Herausforderungen in quantitativer und inhaltlicher Hinsicht auf die Kindertageseinrichtungen zukommen würden. Auf der anderen Seite hat sich das Feld jedoch mit großem Engagement auf die Aufgabe eingelassen, die Kinder in die Kitas zu integrieren. Selbst zu einem Zeitpunkt, als auf der strukturellen Ebene noch wenig systematische Unterstützung bereitgestellt wurde, haben die einzelnen Kitas kreative und pragmatische Lösungen gefunden, mit der Situation umzugehen, die sich ihnen vor Ort darbot. Dabei ist sicherlich zu berücksichtigen, dass nicht alle Herausforderungen für alle Kitas zur Gänze neu waren, sondern manche Kitas bereits auf eine langjährige Erfahrung im Umgang mit kultureller und

17 Leyendecker, B., Kinder mit Fluchterfahrungen - Chancen zur frühen Integration, KiTa NRW, (11) 2016, S. 238. 
sprachlicher Vielfalt aufbauen konnten, während sich andere einer neuen Situation gegenüber sahen. Für viele Einrichtungen erwies sich vor allem die Zusammenarbeit und Verständigung mit den Eltern der betreuten Kinder als schwierig. Typische Probleme betrafen Sprachbarrieren, fehlendes Wissen der Eltern über Routinen und Regeln der Kita, aber auch Unsicherheiten auf Seiten der Fachkräfte bezüglich kultureller Unterschiede. ${ }^{18}$ Oft ließen sich die anfänglichen Hürden überwinden. Gute Erfahrungen machten Kitas mit der Einbeziehung von SprachmittlerInnen oder anderen Vertrauenspersonen der Eltern, wie ehrenamtlichen HelferInnen, Eltern anderer Kita-Kinder oder Menschen der jeweiligen Community, die die Eltern in der Kommunikation mit der Kita unterstützten. Auf diese Weise konnte, ebenso wie über Einladungen zur Hospitation, die enge Einbindung der Eltern in der Eingewöhnungsphase, interkulturelle Feste oder eine bildgestützte Kommunikation, die erforderliche Vertrauensbasis und Transparenz hergestellt werden.

Relativ unterschiedliche Erfahrungen liegen hinsichtlich des Ausmaßes vor, in dem Kitas mit traumatisierten Kindern konfrontiert sind. Ein Teil der Einrichtungen weist hier auf ein eklatantes Defizit in den Möglichkeiten einer psychologischen Betreuung der Kinder, aber auch auf fehlende Beratungsmöglichkeiten für ErzieherInnen hin. Für andere Einrichtungen ist das Thema von geringerer Relevanz. Selbst wenn ein (kleiner) Teil der schutzsuchenden Kinder traumatische Erfahrungen mitbringt, scheinen diese im Kita-Alltag keine beherrschende Rolle zu spielen. Vielmehr werden die Kinder von den Fachkräften häufig als resilient wahrgenommen. ${ }^{19}$

\section{Unterstützung und Vernetzung: Der Bedarf der Kitas ist hoch}

Im Umgang mit den Herausforderungen durch diese neue AdressatInnengruppe haben die Fachkräfte vielfach Unterstützungsbedarfe kommuniziert, die ganz unterschiedlichen Bereichen zuzuordnen sind. So wurden bspw. in der DJI-Kitabefragung Unterstützungsbedarfe mit Blick auf die sprachlichen Hürden, die personelle Ausstattung, pädagogische Fragen und Informationen zu rechtlichen Rahmenbedingungen benannt. ${ }^{20}$ Vor allem hinsichtlich der Verbesserung der Kommunikation mit den Eltern wurden die Kitas dabei unter anderem durch DolmetscherInnen oder SprachmittlerInnen, aber auch durch die Vermittlung von Deutschkursen für Eltern unterstützt. Die Bereitstellung von Informationsmaterialien oder spezifischen Fortbildungen sind weitere Bereiche, in denen die Kitas bereits frühzeitig Hilfe erhalten haben. Hingegen fehlt ihnen vor allem zusätzliches Personal für die Arbeit mit den geflüchteten Kindern und ihren Familien.

Deutlich wurden hinsichtlich der vorhandenen und zum Teil fehlenden Unterstützung vor allem zwei Aspekte: Vielfach wurden Hilfen nicht nur durch die klassischen Akteure im System der Kindertagesbetreuung zur Verfügung gestellt wie den Trägern, den Kommunen oder den Ländern, sondern auch Ehrenamtliche und lokale Helferkreise unterstützen die Kitas bei der Bewältigung der Aufgaben - in einem bisher ungekannten Ausmaß. In der DJI-Studie konnte

18 Baisch et al. (Anm. 10), S. 44.

19 Konferenz der evangelischen und katholischen Kirchenleitungen Baden-Württemberg und ihrer Spitzen/ Trägerverbände über Kindergartenfragen (Hrsg.), Frühkindliche Förderung von Kindern aus Flüchtlingsfamilien, unveröff. Manuskript 2014.

20 Baisch et al. (Anm. 10), S. 58. 
mehr als jede dritte Kita auf das Engagement von Ehrenamtlichen zählen. ${ }^{21}$ Damit gewann eine Gruppe in der Arbeit der Kitas an Bedeutung, die in anderen Feldern der Kinder- und Jugendhilfe bereits vielfach involviert ist, in der Kindertagesbetreuung aber keine nennenswerte Rolle spielte. Nimmt man die MitarbeiterInnen der Asylfachdienste, Kirchengemeinden, Vereine und sonstige Helfer hinzu, so sind hier für die Kitas neue Kooperationsbeziehungen und -strukturen entstanden, von denen nicht nur geflüchtete Kinder, sondern auch ihre Familien in vielfacher Weise profitieren.

Der zweite zentrale Aspekt, der deutlich wird, ist, dass strukturelle Probleme, die das Feld der Kindertagesbetreuung bereits vor der hohen Zuwanderung schutzsuchender Familien kennzeichneten, wie fehlende Betreuungsplätze, Knappheit bei den Personalressourcen und in der Folge fehlende zeitliche Ressourcen für die Förderung der Kinder, sich noch weiter verschärft haben. Sie stellen eine gelingende Integration schutzsuchender Kinder jedoch infrage, da diese nicht nur gleiche Zugangschancen, sondern auch eine hohe Qualität der Bildung, Betreuung und Erziehung voraussetzt.

Wie der Beitrag zu zeigen versucht hat, wird trotz abgeschwächter Fluchtmigration die Aufgabe, den Zugang zu Einrichtungen der Kindertagesbetreuung zu erleichtern und einen qualitativ guten Rahmen für die Förderung von Kindern aus schutzsuchenden Familien zu gewährleisten, längerfristig bedeutsam bleiben. Während sich Kitas dabei vielfach auf einen starken Rückhalt in der Zivilgesellschaft stützen können, gilt es jedoch auch eine andere Tendenz im Auge zu behalten. Es bleibt zu beobachten, ob und wie die in Teilen der Gesellschaft zunehmende Fremdenfeindlichkeit sich auf die Integration schutzsuchender Kinder in den Kitas auswirkt und welche Unterstützung Fachkräfte brauchen, um solchen Tendenzen entgegentreten zu können.

Verf.: Birgit Riedel, Deutsches Jugendinstitut e.V., Nockherstraße 2, D-81541 München, EMail:riedel@dji.de

Christiane Meiner-Teubner, Technische Universität Dortmund, Fakultät 12 - Erziehungswissenschaft und Soziologie, Forschungsverbund DJI/TU Dortmund, Arbeitsstelle Kinder- und Jugendhilfestatistik, Vogelpothsweg 78,D-44227 Dortmund, E-Mail: christiane.meiner@tu-dortmund.de

21 Ebd., S. 53. 